\title{
GLOSSARY OF ARABIC TERMS
}

\author{
${ }^{c} \bar{a} d a \quad$ customary law, tradition \\ adhān (mu'adhdhin) call to prayer (Muezzin) \\ abl people \\ ${ }^{c} \bar{a}$ lim, pl. ${ }^{c}$ ulam $\bar{a}^{-} \quad$ religious scholar \\ amìr \\ commander (amir al-müminin: commander of \\ the faithful) \\ ansār supporters \\ ${ }^{c}$ aqida confession (of the faith), catechetic theology, \\ doctrine \\ ${ }^{c} a s \underline{r} \quad$ afternoon prayer \\ ${ }^{c}$ awrah intimate (parts), pudenda \\ baraka blessing power \\ bātin \\ hidden, inner meaning \\ $b^{c} d^{c} a$, pl. $b i d a^{c}$ \\ (un-Islamic) innovation \\ $b i k \bar{a}^{\circ}$ \\ wailing, weeping \\ $d \bar{a}^{3}$ ira \\ recitation/study circle \\ dalīl, pl. dalāìil \\ guide \\ $d \bar{a} r$ \\ land, house \\ $d a^{c} w a$ \\ cause, message, propaganda ( $d \bar{a}^{c} i y a$ : a person \\ who represents a cause, pl. $\left.d u^{c} a h\right)$ \\ dhikr \\ Sufi meditation exercises, ceremony, ritual




\begin{tabular}{|c|c|}
\hline $\operatorname{din}$ & religion \\
\hline diya & blood money \\
\hline$d u^{c} \bar{a}^{\nu}$ & supplication, supererogatory prayer \\
\hline fajr $(s ̦ b b ̣)$ & morning (prayers) \\
\hline fatwā, pl. fatāwa & formal (yet, not binding) legal opinion \\
\hline fiqh (usül al-)fiqh & law, jurisprudence (Islamic legal theory) \\
\hline fitna & chaos, disorder, disunity \\
\hline ghawth & succour (ghawth al-zamān: succour of the age) \\
\hline ḅadd, pl. budūd & qur ānic regulations and punishments \\
\hline badith, pl. aḅädith & the (discipline of) Prophetic traditions \\
\hline ḅajj (ḅajjj) & pilgrimage (pilgrim) \\
\hline ḅaqiqa & truth \\
\hline b̧araka & $\begin{array}{l}\text { movement, speed (in terms of the rules for the } \\
\text { recitation of the Qur }{ }^{\circ} \bar{a} \text {, tajwid) }\end{array}$ \\
\hline bawāla & cheque, bill of exchange, money transfer \\
\hline bijāb & amulet, protection, veil \\
\hline hijra & (e)migration \\
\hline hilāl & new moon, crescent \\
\hline birāba & robbery \\
\hline bisba & $\begin{array}{l}\text { guarding (public order), (mubtasib: a person who } \\
\text { guards) }\end{array}$ \\
\hline b̧izb, pl. $a b ̣ z \bar{a} b$ & $\begin{array}{l}\text { group, part (one of sixty recitational sections of } \\
\text { the Qurª̄n) }\end{array}$ \\
\hline${ }^{c} i b \bar{a} d \bar{a} t(\mathrm{pl})$. & acts of devotion, religious obligations \\
\hline ibtila $\bar{a}^{-}$ & trial, visitation \\
\hline${ }^{c} \bar{\imath} d$, pl. $a^{c} y \bar{a} d$ & $\begin{array}{l}\text { festival day (such as }{ }^{c} \bar{i} d a l-k a b i r l a l-a d b \bar{a} \text { and }{ }^{c} \bar{i} d \\
a l \text {-fttr) }\end{array}$ \\
\hline$i b y \bar{a}^{-}$ & revivication, revival \\
\hline$i j \bar{a} z a$ & authorisation (to represent and/or to teach a text) \\
\hline ijtiḅa àd & effort \\
\hline$i k h w a \bar{n}$, sg. akh & $\begin{array}{l}\text { brothers (al-ikhwān al-muslimün: 'the Muslim } \\
\text { Brothers') }\end{array}$ \\
\hline${ }^{c}$ ilm, pl. ${ }^{c}$ ulüm & discipline (of learning), science \\
\hline cilm al-falak & astrology, numerology, astronomy \\
\hline ìmām & leader (of prayers) \\
\hline
\end{tabular}




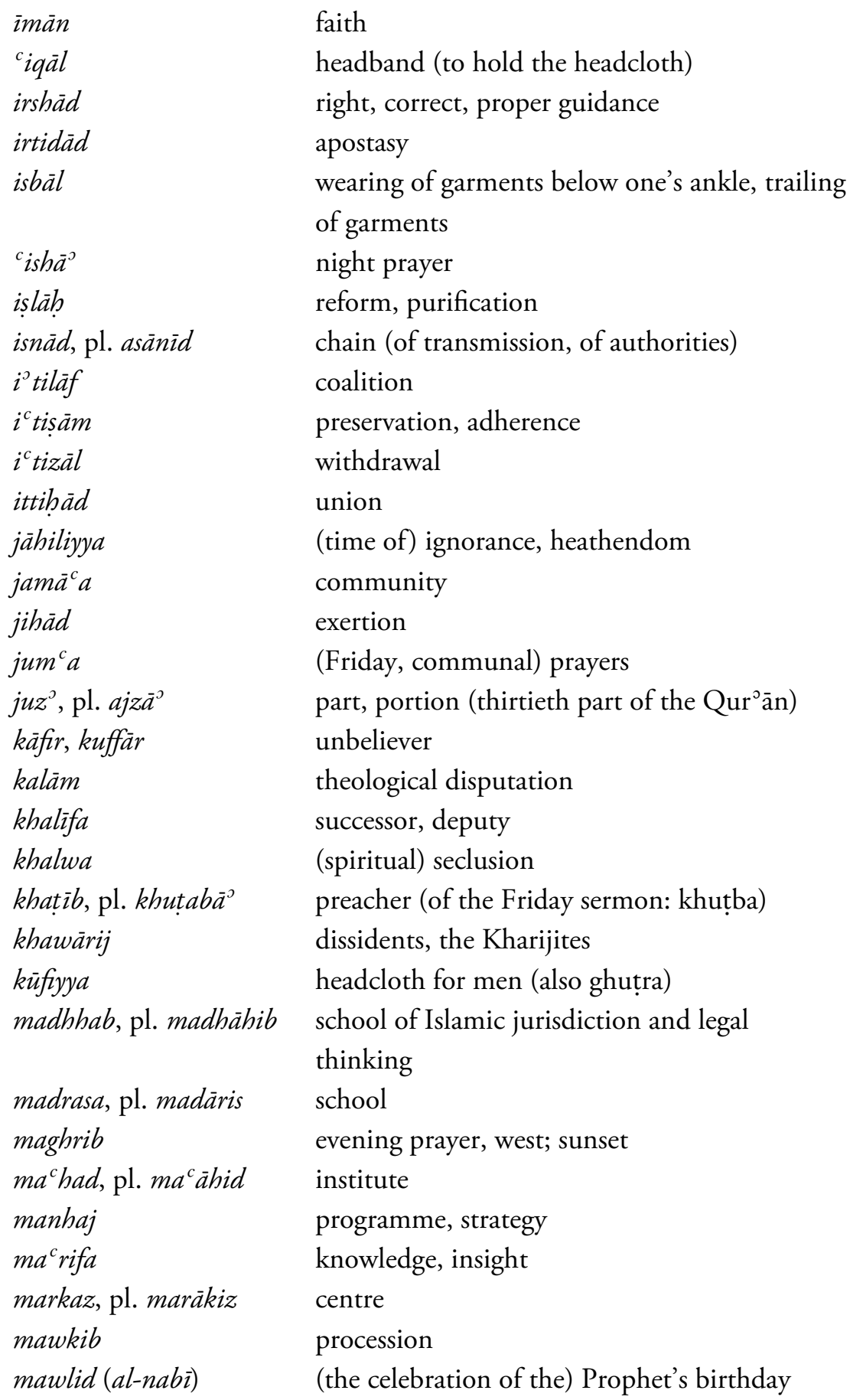




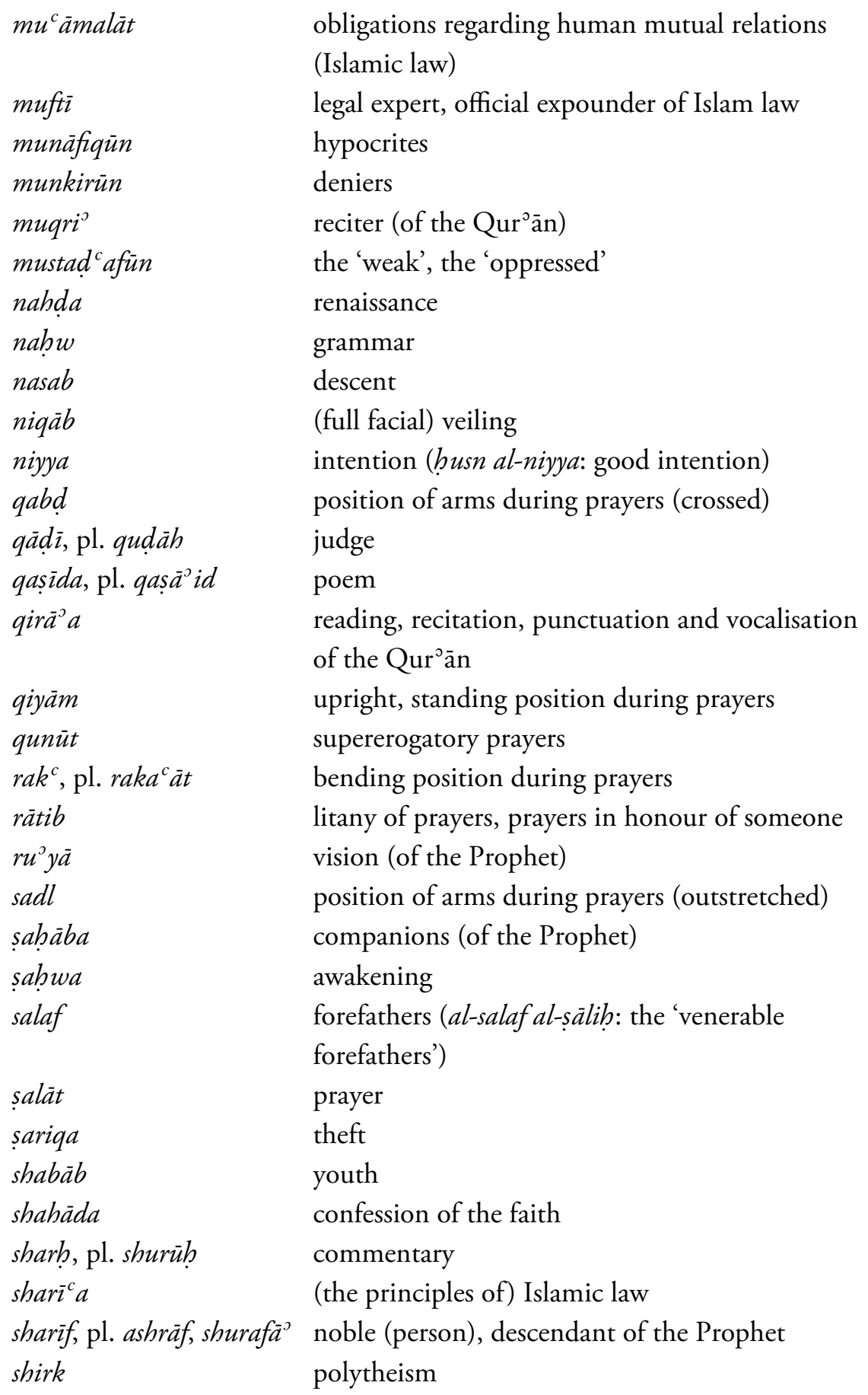




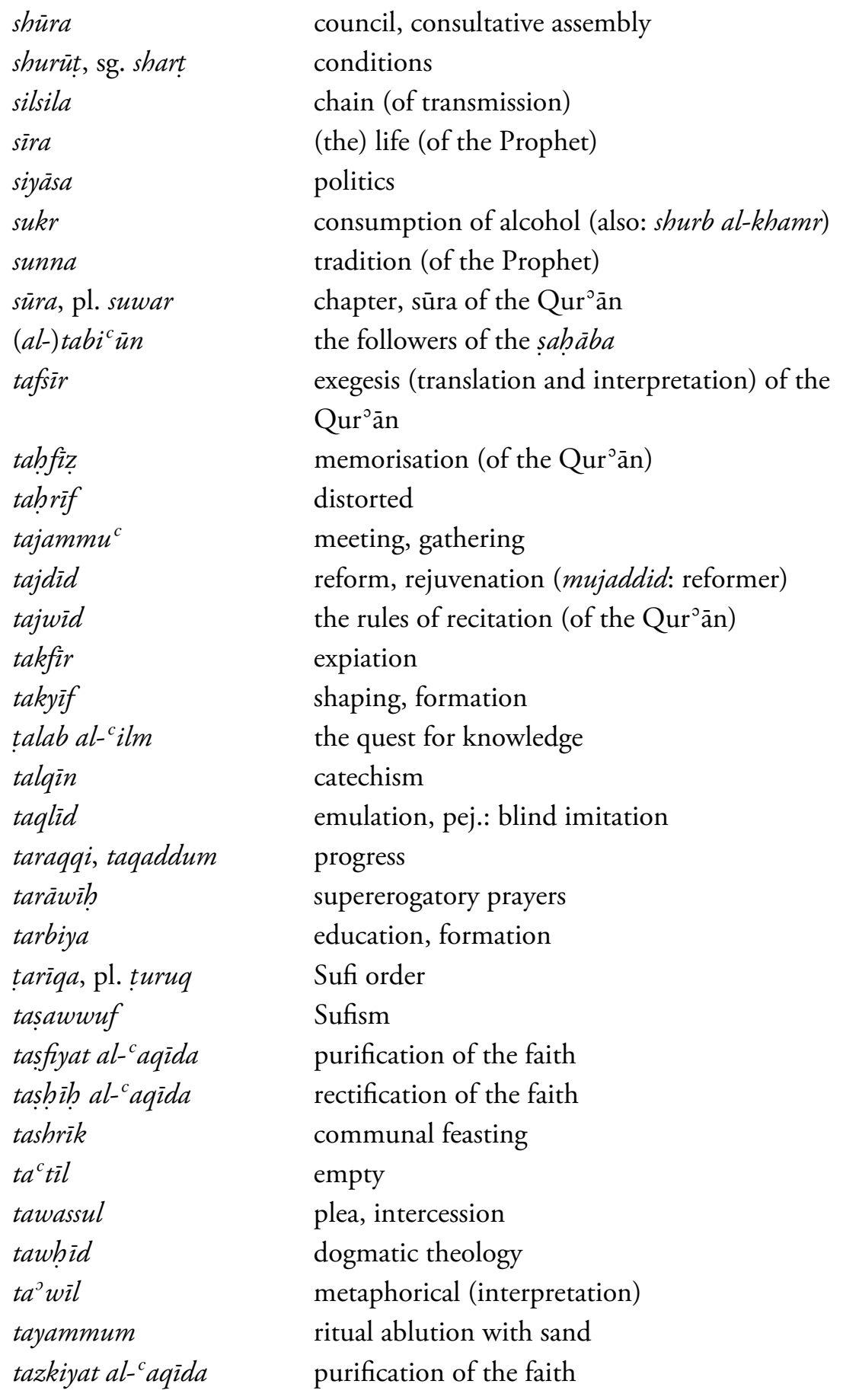




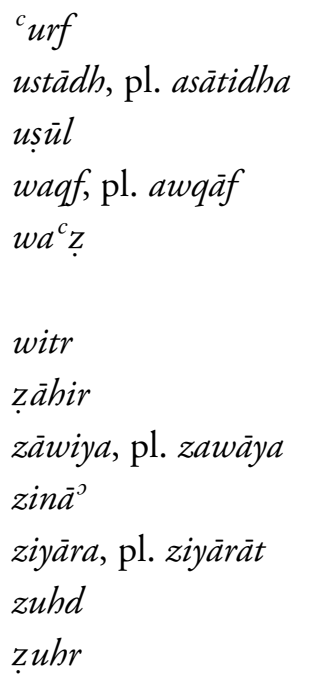

customary law, tradition

professor

the basics (of faith, for instance)

charitable foundation

exhorting sermon (the person who exhorts is a $w \bar{a}^{c} i z$, pl. $\left.w u^{c c} a z\right)$

supererogatory prayers

outward, visible

Sufi convent, centre

extramarital sex

local pilgrimage, shrine pilgrimage

ascetism

noon prayer 